\title{
Field Effect and Light-Assisted a-Si:H Sensors for Detection of Ions in Solution
}

\author{
J. Costa ${ }^{1, *}$, M. Fernandes ${ }^{1}$, M. Vieira ${ }^{1}$, G. Lavareda ${ }^{2}$, C. N. Carvalho ${ }^{3}$, and A. Karmali ${ }^{4}$ \\ ${ }^{1}$ Electronics Telecommunication and Computer Dept. ISEL, R.Conselheiro Emídio Navarro, 1949-014 Lisboa, Portugal \\ ${ }^{2}$ CTS-UNINOVA, Monte da Caparica 2829-516, Portugal \\ ${ }^{3}$ ICEMS-Av. Rovisco Pais, Lisboa, Portugal \\ ${ }^{4}$ Chemical Engineering and Biotechnology Research Center, Instituto Superior Engenharia de Lisboa R.Conselheiro Emídio Navarro, \\ 1949-014 Lisboa, Portugal
}

(Received: 6 June 2009. Accepted: 29 January 2010)

\begin{abstract}
In this paper we present an amorphous silicon device that can be used in two operation modes to measure the concentration of ions in solution. While crystalline devices present a higher sensitivity, their amorphous counterpart present a much lower fabrication cost, thus enabling the production of cheap disposable sensors for use, for example, in the food industry. The devices were fabricated on glass substrates by the PECVD technique in the top gate configuration, where the metallic gate is replaced by an electrolytic solution with an immersed $\mathrm{Ag} / \mathrm{AgCl}$ reference electrode. Silicon nitride is used as gate dielectric enhancing the sensitivity and passivation layer used to avoid leakage and electrochemical reactions. In this article we report on the semiconductor unit, showing that the device can be operated in a light-assisted mode, where changes in the $\mathrm{pH}$ produce changes on the measured ac photocurrent. In alternative the device can be operated as a conventional ion selective field effect device where changes in the $\mathrm{pH}$ induce changes in the transistor's threshold voltage.
\end{abstract}

Keywords: Amorphous Silicon, LAPS, ISFET.

\section{INTRODUCTION}

This paper reports our efforts in the development of an ion sensitive sensor based on hydrogenated amorphous silicon. Among various sensor requirements, there is often interest in making compact and inexpensive sensors thus the interest in using amorphous silicon.

The hydrogenated amorphous silicon (a-Si:H) technology allows the fabrication of a broad range of devices, such as thin film transistors (TFT) and photodiodes, on a variety of low cost rigid or flexible substrates, like glass metal foil or polymeric films. Additionally, the low-temperature processing and the good optoelectronic properties makes a-Si:H an attractive material for the development of low cost sensors for the detection of small quantities of biological molecules. In the past, these devices have been used for the detection of hydrogen in the gas phase using a MISFET structure with a palladium gate, which acts as a catalyzer for the dissociation of the molecular hydrogen. ${ }^{1}$ Among others, Gotoh et $\mathrm{al}^{2}{ }^{2}$ reported the use of a-Si:H as the active material

${ }^{*}$ Corresponding author; E-mail: jcosta@ deetc.isel.ipl.pt in an Ion Selective Field Effect Transistor (ISFET) for biosensing applications in an electrolyte medium. Since then, changes in the gate voltage at fixed source-drain voltage and source-drain current, caused by changes in ion concentration have been thoroughly investigated using various dielectric gate materials. ${ }^{3}$

This paper is mainly concerned with the semiconductor unit which will be described in the next section. Tests have been made to show that the unit is sensitive to the $\mathrm{pH}$. Various studies have also shown that is possible to adapt ISFET devices using ion selective membranes and immobilized enzymes to make them sensitive to various types of ions. ${ }^{4}$ In fact our long term goal is to build a sensor of toxic amides, which is disposable, thus the interest in using an inexpensive technology such as amorphous silicon. Nevertheless its performance should be comparable to its crystalline counterpart thus making it an interesting solution.

\section{DEVICE FABRICATION}

Due to the operation principle of the ISFET the top gate configuration was chosen, the structure of the device is 


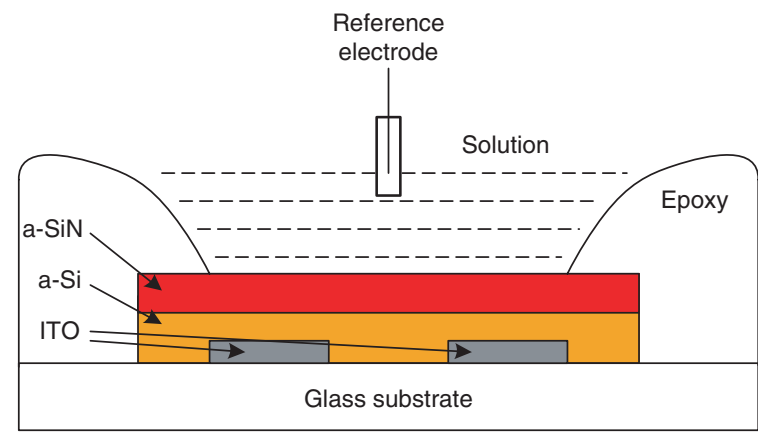

Fig. 1. a-Si:H ISFET structure.

Table I. Fabrication conditions.

\begin{tabular}{lcc}
\hline Material & a-Si:H & a-SiN \\
\hline Substrate temperature $\left({ }^{\circ} \mathrm{C}\right)$ & 300 & 350 \\
Gas pressure (mTorr) & 163 & 172 \\
RF power $(W)$ & 20 & 20 \\
Silane flow (SCCM) & 20 & 16 \\
Ammonia flow (SCCM) & - & 4 \\
\hline
\end{tabular}

sketched in Figure 1. The source and drain contacts were patterned on an Indium Thin Oxide (ITO) covered glass, defining a channel whose dimensions are: Width $(W)=$ $3500 \mu \mathrm{m}$ and Length $(L)=100 \mu \mathrm{m}$ thus with a geometric factor $W / L=35$. Next, the semiconductor and insulator layers were fabricated sequentially by plasma-enhanced chemical vapor deposition (PECVD) under the deposition conditions shown in Table I. The resulting thicknesses are $1200 \AA$ and $2000 \AA$ for the a-Si:H and a-SiN layers respectively. The final step, prior to encapsulation, was the patterning of the dual layer in order to expose the source and drain contacts. The device was then encapsulated using epoxy resin leaving exposed only the a-SiN pH-sensitive surface over the channel region.

\section{I-V CHARACTERISTICS}

After assembling, the devices were tested by measuring the $I_{\mathrm{D}}-V_{\mathrm{DS}}$ and $I_{\mathrm{D}}-V_{\mathrm{GS}}$ as in conventional MOSFET's. The operation of the devices is very similar to conventional transistors, except for the fact that metal gate is replaced by a buffer solution and a reference electrode, as presented in Figures 1 and 2. By dipping the device and the reference electrode $(\mathrm{Ag} / \mathrm{AgCl})$ in a buffer solution with known

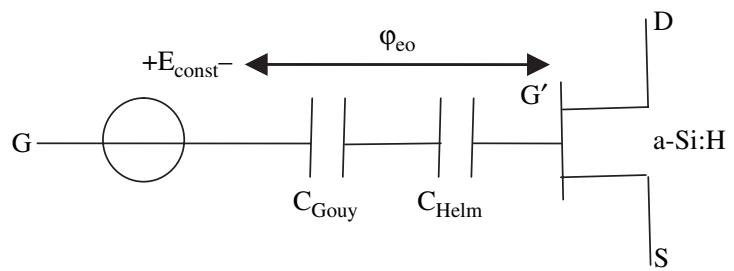

Fig. 2. Equivalent circuit of the ISFET device, showing the capacitors and potentials described in the text.

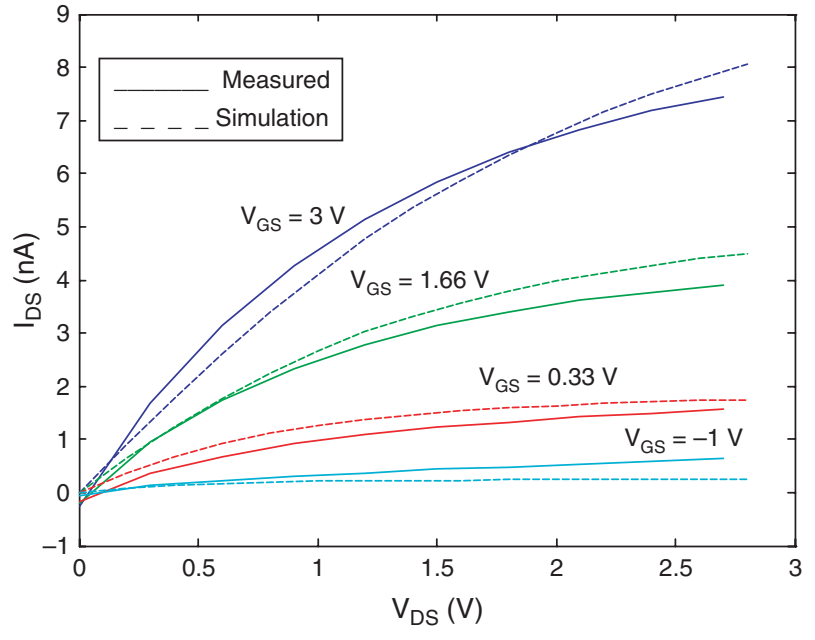

Fig. 3. $I_{\mathrm{DS}}-V_{\mathrm{DS}}$ charactreristics for $\mathrm{pH} 7$ and different $V_{\mathrm{GS}}$ values.

$\mathrm{pH}$ and stepping the voltage applied to the drain and reference electrode the curves can be obtained, as presented in Figure 3. Apart from the low current levels observed, and the comparatively high off currents, the devices shows good field effect behavior with a threshold voltage $\left(V_{\mathrm{t}}\right)$ around $-1 \mathrm{~V}$ consistent with the slightly $n$-type behavior of the intrinsic a-Si:H semiconductor material. ${ }^{5}$

\section{ELECTRICAL MODEL}

An electrical model was used to obtain insight into device operation. We have used a methodological approach inspired in the behavioral model of Martinoia and Massobrio for crystalline ISFETs, which was adapted to the a-Si:H transistor studied here. The SPICE simulation was performed using the software AIM-SPICE. ${ }^{6}$

In ISFETs the threshold voltage provides a mean to detect the ion concentration in solution. The gate voltage is the voltage of the reference electrode, so the threshold voltage $V_{\text {th }}^{\text {ISFT }}$ contains terms which reflect the presence of the electrolyte, ${ }^{7}$ in particular $\varphi_{\mathrm{eo}}$ which depends on the ion concentration. It is this term that makes the ISFET sensitive to the solution $\mathrm{pH}$.

We have considered the ISFET divided in two stages: an electronic stage, the a-Si:H transistor, and an electrochemical stage which includes the capacitances of the double layer and the interface potentials of the reference electrode (see Fig. 2). From the site-binding theory we have a relation between the proton concentration $H_{\mathrm{s}}$ and the surface charge density $\sigma_{0}$, at the electrolyte-insulator interface. ${ }^{8}$ For the $\mathrm{Si}_{3} \mathrm{~N}_{4}$ insulator the expression is the following:

$$
\sigma_{0}=q N_{\mathrm{Sil}}\left(\frac{H_{\mathrm{S}}^{2}-K_{\mathrm{A}} K_{\mathrm{B}}}{H_{\mathrm{S}}^{2}+K_{\mathrm{A}} H_{\mathrm{S}}+K_{\mathrm{A}} K_{\mathrm{B}}}\right)+q N_{\mathrm{Nit}}\left(\frac{H_{\mathrm{S}}}{H_{\mathrm{S}}+K_{\mathrm{N}}}\right)
$$

where $q$ is the electron charge; $K_{\mathrm{N}}$ the dissociation constant for the amine sites; $K_{\mathrm{A}}, K_{\mathrm{B}}$ the two dissociation 
constants for silanol sites; $N_{\mathrm{Nit}}$ and $N_{\mathrm{Sil}}$ the surface densities of primary amine and silanol sites, respectively. The Boltzmann factor provides the relation between the bulk proton concentration $\left(H_{\mathrm{b}}\right)$ and the interface proton concentration $\left(H_{\mathrm{s}}\right)$ :

$$
H_{\mathrm{s}}=H_{\mathrm{b}} \exp \left(-\varphi_{\mathrm{eo}} / V_{\mathrm{T}}\right)
$$

where $\varphi_{\text {eo }}$ is the electrolyte-insulator interface potential.

On the other hand, from the electrical double layer theory, and assuming, as usual, that the charge density in the semiconductor is much smaller than $\sigma_{0},{ }^{9,10}$ we have the following relation:

$$
\sigma_{0}=C_{\mathrm{eq}} \varphi_{\mathrm{eo}}
$$

where $C_{\mathrm{eq}}$ is the series capacitance formed by the GouyChapman and Helmholtz capacitances:8,9

$$
\begin{gathered}
C_{\mathrm{Helm}}=\frac{\varepsilon_{\mathrm{IHP}} \varepsilon_{\mathrm{OHP}}}{\varepsilon_{\mathrm{OHP}} d_{\mathrm{IHP}}+\varepsilon_{\mathrm{IHP}} d_{\mathrm{OHP}}} W L \\
C_{\text {Gouy }} \cong \frac{\sqrt{8 \varepsilon_{\mathrm{w}} k T}}{2 V_{\mathrm{T}}}
\end{gathered}
$$

In the expressions, $W$ and $L$ are the ISFET channel width and length; $\varepsilon_{\mathrm{OHP}}$ and $\varepsilon_{\mathrm{IHP}}$ the dielectric constants of the outer and inner Helmoholtz layers, $d_{\mathrm{OHP}}$ and $d_{\mathrm{IHP}}$ the length of the inner and outer Helmholtz layers, $\varepsilon_{\omega}$ the dielectric constant of the electrolyte, $T$ the temperature, $k$ the Boltzmann constant and $V_{\mathrm{T}}=k T / q$ the thermal voltage.

When Eqs. (2) and (3) are inserted into Eq. (1) the result is a non-linear equation in $\varphi_{\text {eo }}$ which is dependent on proton concentration $(\mathrm{pH})$ and density of binding sites $N_{\text {Nit }}$ and $N_{\text {Sil }}$. The equation can be solved numerically to determine the value of $\varphi_{\text {eo }}$ to be used in the equivalent circuit of Figure 2. The electrochemical parameters, such as number of binding sites have been taken from the literature. ${ }^{7}$

For the a-Si:H stage we have used the model developed by Schur et al., ${ }^{11}$ with the physical dimensions set according to our device and the remaining parameters extracted from the experimental curves using the tool AIM-extract. ${ }^{6}$ The fitting was reasonably successful as can be seen in Figure 3. More details about the fitting methodology and its limitations can be found in Cerdeira et al. ${ }^{12}$ The SPICE simulation was performed using the software AIMSPICE. ${ }^{6}$

\section{FIELD EFFECT OPERATION}

In this mode of operation a bias voltage is applied between the drain and source $\left(V_{\mathrm{DS}}\right)$ of the transistor and the drain current $\left(I_{\mathrm{DS}}\right)$ is measured as a function of the voltage between the reference electrode and the source $\left(V_{\mathrm{GS}}\right)$. Changes in $\mathrm{pH}$ affect the $V_{\mathrm{t}}$ of the transistor and therefore should produce changes in the $I_{\mathrm{DS}}-V_{\mathrm{GS}}$ characteristic.

In Figure 4 we compare the experimental $I_{\mathrm{DS}}-V_{\mathrm{GS}}$ characteristic for two different $\mathrm{pH}$ levels. The sensitivity

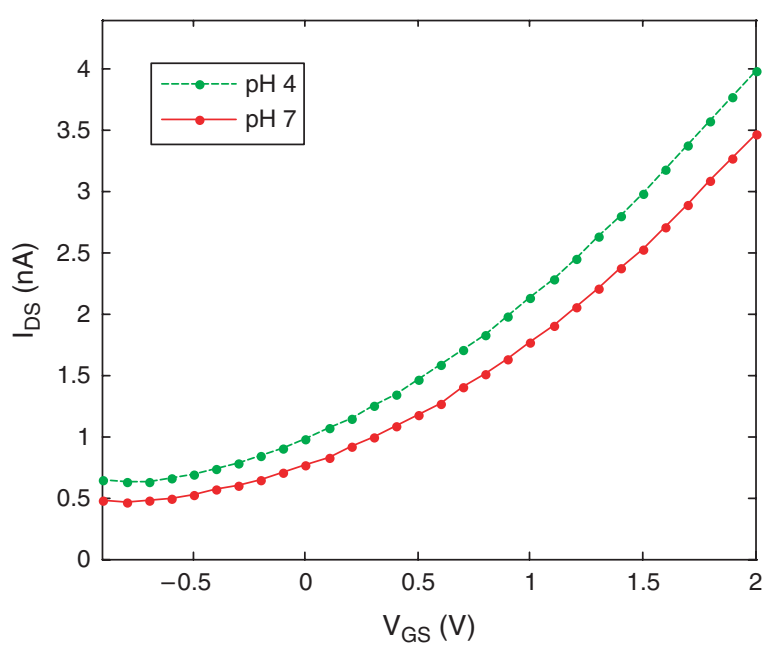

Fig. 4. The current-voltage characteristics $\left(I_{\mathrm{DS}}\right.$ vs. $\left.V_{\mathrm{GS}}\right)$ for two $\mathrm{pH}$ levels.

calculated from the curves is close to the expected Nernstian behavior and indicates that the device can operate as a conventional ISFET device.

\section{LIGHT-ASSISTED OPERATION}

The working principle of solid state electronic $\mathrm{pH}$ sensors relies on the modification of the surface potential caused by the concentration of charged ions in the solution. The use of this effect in field effect transistors is straightforward, and can be accounted by including in the determination of the threshold voltage some extra terms related to the extra interfaces/layers and a term dependent on the ion concentration as explained in the previous section. Nevertheless, other techniques that explore the surface potential can be applied to construct $\mathrm{pH}$ sensors. One of the techniques, widely used in other fields, uses the variation of the depletion layer formed in the semiconductor for the determination of the surface potential in semiconductorinsulator structures. This technique is used on a number of sensors ${ }^{13,14}$ where the depletion layer width is

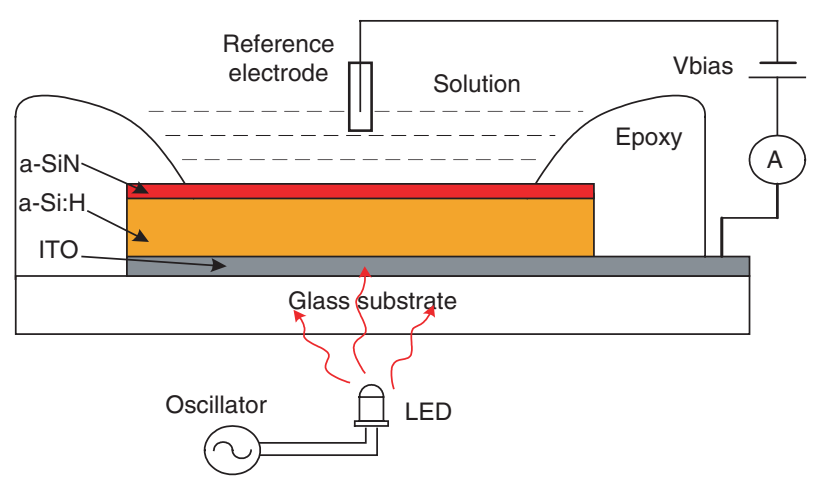

Fig. 5. Schematic of the light-assisted operation mode showing the modulated light source and the Bias Voltage. 


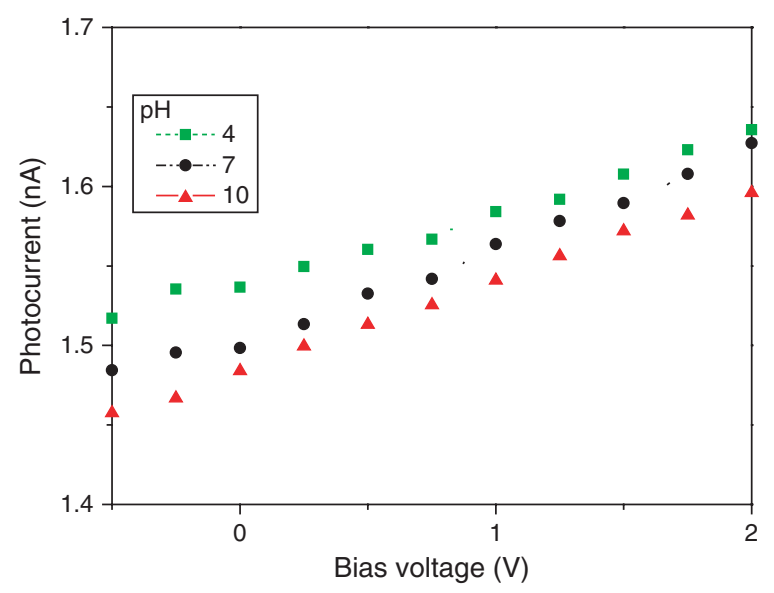

Fig. 6. Photocurrent as a function of Bias Voltage and $\mathrm{pH}$ level. The results were obtained for a Bias Voltage is in the range $-1 \mathrm{~V}-2 \mathrm{~V}$.

probed by measuring the ac photocurrent induced by a modulated light source. When this technique is applied to an electrolyte-insulator-semiconductor structure the $\mathrm{pH}$ of the electrolyte can be detected resulting in a lightaddressable potentiometric sensor (LAPS). ${ }^{15}$ In this work we use the LAPS technique applied to an amorphous silicon based electrolyte-insulator-semiconductor (EIS) structure. Figure 5 shows the schematic of this operation mode.

Figure 6 shows the photocurrent as a function of Bias Voltage and $\mathrm{pH}$ level for a $75 \mathrm{~Hz}$ modulated light source of $650 \mathrm{~nm}$. The acidic solution has higher photocurrent than the alkaline solution at the same bias voltage, thus the results indicate that the response of the device is dependent of the $\mathrm{pH}$. Further investigation is needed to determine the modulation frequency dependence.

\section{CONCLUSIONS}

The overall aim of our research is to produce a low cost ion sensitive transistor that can be coupled to a membrane, thus the interest in amorphous silicon. The results indicate the possibility of using the a-Si:H device as an ion sensitive sensor in two operation modes. The $I_{\mathrm{DS}}-V_{\mathrm{GS}}$ curve is dependent of the $\mathrm{pH}$ of the solution, thus the device can be operated as a conventional ISFET device. In addition it can also be operated in a light assisted mode, where the drain and source are short-circuited and a bias voltage is applied between the drain and reference electrode. In this case changes in the $\mathrm{pH}$ can be detected through changes in the ac photocurrent induced by a modulated light source.

Future work will be necessary to evaluate the sensor at different temperature and proton concentrations. Further studies will focus on the geometry of the device to increase drain currents and also on its integration with the biological sensing unit to obtain ion selectivity.

\section{References and Notes}

1. Lundström, C. Svensson, A. Spetz, H. Sundgren, and F. Winquist, Sens. Actuators, B 13-14, 16 (1993).

2. M. Gotoh, S. Oda, I. Shimizu, A. Seki, E. Tamiya, and I. Karube, Sens. Actuators 16, 55 (1989).

3. L. Mariucci, G. Fortunato, A. Pecora, A. Bearzotti, P. Carelli, and R. Leoni, Sens. Actuators, B 6, 29 (1992).

4. M. L. Hamlaoui, R. Kherrat, M. Marrakchi, N. JaffrezicRenault, and A. Walcarius, Mater. Sci. Eng., $C$ 21, 25 (2002).

5. R. Schropp and M. Zeman, Amorphous and Microcrystalline Silicon Solar Cells: Modeling, Materials, and Device Technology, Kluwer Academic Publisher (1998).

6. T. A. Fjeldly, T. Ytterdal, and M. Shur, Introduction to Device Modeling and Circuit Simulation, John Wiley and Sons, New York (1998).

7. S. Martinoia, G. Massobrio, and L. Lorenzelli, Sens. Actuators, B 105, 14 (2005).

8. D. E. Yates, S. Levine, and T. W. Healy, J. Chem. Soc. Farady Trans. 70, 1807 (1974).

9. S. Martinoia and G. Massobrio, Sens. Actuators, B 62, 182 (2000).

10. L. Bousse, N. F. de Rooij, and P. Bergveld, IEEE Trans. Electron Devices ED 30, 1263 (1983).

11. M. Shur and M. Hack, J. Appl. Phys. 55, 3831 (1984).

12. A. Cerdeira, M. Estrada, R. Garcia, A. Ortiz-Conde, and F. J. Garcia Sanchez, Solid-State Elctronics 45, 1077 (2001).

13. M. Fernandes, M. Vieira, and R. Martins, J. Non-Cryst. Solids 352, 1801 (2006).

14. M. Nakao, T. Yoshinobu, and H. Lwasaki, Sens. Actuators, B 20, 119 (1994).

15. D. G. Hafeman, J. W. Paree, and H. M. McConnell, Science 240,1182 (1988). 